\title{
II. Abkürzungen der Zeitschriften
}

Die Nummern beziehen sich auf den ausführlichen Titel der Zeitschriften und Veröffentlichungen in Liste III S. X und Liste IV S. XIII. Ist anstatt einer Nummer ein $†$ gesetzt, so ist die betreffende Abkürzung in AJB 45 S. XI einzusehen.

\begin{tabular}{|c|c|c|c|}
\hline & $\mathrm{Nr}$. & & \\
\hline$A(a), A A(b), A A(c) \ldots \ldots \ldots$ & $\mathbf{3}$ & Mem SA It & \\
\hline$\ldots \ldots \ldots \ldots \ldots \ldots \ldots \ldots$ & $\dagger$ & MN ASSA & \\
\hline$\ldots \ldots \ldots \ldots \ldots \ldots$ & $t$ & $\mathrm{MN} \ldots \ldots$ & \\
\hline$\ldots \ldots$. & $t$ & MN Geophys Suppl .. & \\
\hline nn d'Astrophys . . . . . . . & 5 & $N C$ & \\
\hline an d Hydrog $\ldots \ldots \ldots \ldots \ldots$ & 4 & $\mathbf{T}$ & \\
\hline$\ldots \ldots \ldots \ldots \ldots$ & $\dagger$ & Nat & \\
\hline s Mat Astr Fys $\ldots \ldots \ldots \ldots$ & 7 & urwiss ... & \\
\hline r Circ USSR ........... & & & \\
\hline$\ldots \ldots \ldots \ldots \ldots$ & 15 & & \\
\hline$\ldots \ldots \ldots \ldots \ldots$ & & s Ber & \\
\hline$\ldots \ldots \ldots \ldots$ & & Phys Rev. & \\
\hline$\ldots \ldots \ldots \ldots$ & 171 & Astr . & \\
\hline & & Roy Soc & \\
\hline . & & AAS $\ldots .$. & \\
\hline$\ldots 1$ & 122 & l ASP & \\
\hline & 8 & $\mathrm{Ci}$ & \\
\hline ... & 17 & k Mitt. & \\
\hline & 21 & Pulk Publ . & \\
\hline$\ldots$ & 22 & 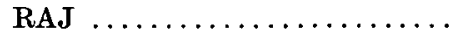 & \\
\hline$\ldots$ & 2 & V Ast & \\
\hline$n$ & 84 & $\mathbf{v}$ Mo & \\
\hline$\checkmark$ Bull & 86 & Rev d'Opt Théor et Instr ...... & \\
\hline arv Card & 89 & & \\
\hline arv Circ & 8 & an Publ & \\
\hline$\ldots \ldots \ldots 8$ 87, & & & \\
\hline$\ldots \ldots \ldots \ldots \ldots$ & & $\pi$ & \\
\hline an RAS & + & s Inter Astr Union & \\
\hline$\ldots \ldots \ldots \ldots$ & 27 & Circ $\ldots \ldots \ldots \ldots \ldots \ldots \ldots$ & \\
\hline & & & \\
\hline pt Soc Amer & 32 & $\mathrm{U}$ & \\
\hline Akt Medd & & cel & \\
\hline ck Bull & & & \\
\hline & & h Nat Ac Proc. & \\
\hline & 127 & & \\
\hline $\operatorname{Rer}$ & 51 & & \\
\hline & & & \\
\hline DAA & & & \\
\hline & & & \\
\hline
\end{tabular}

Weitere Zeichen und Abkürzungen, die sich auf Kleine Planeten und Veranderliche beziehen, sind in $\S 56$ bzw. $\$ 85 \mathrm{~S}$. 194 erläutert. 